\title{
Pensamiento Pedagógico en la Enseñanza de las Ciencias. Análisis de las Creencias Curriculares y sus Implicancias para la Formación de Profesores de Enseñanza Media
}

\author{
Saúl A. Contreras \\ Universidad de Santiago de Chile, Facultad de Humanidades, Departamento de Educación, Alameda 3363, \\ Estación Central, Santiago-Chile (e-mail: saul.contreras@usach.cl)
}

Recibido Jun. 18, 2015; Aceptado Ago. 11, 2015; Versión final Oct. 9, 2015, Publicado Feb. 2016

\begin{abstract}
Resumen
El objetivo de este trabajo fue estudiar el pensamiento pedagógico de un conjunto de 303 profesores chilenos que enseñan ciencias, mediante el análisis de las creencias curriculares y creencias de actuación curricular de los profesores. Este estudio es parte de una investigación más amplia (FONEDCYT 11130150) sobre la formación de futuros profesores, que tiene como antecedentes el pensamiento, el conocimiento y la práctica de profesores chilenos que enseñan ciencias en educación media. La información recogida con un cuestionario tipo Likert y tratada con técnicas multifactoriales, muestra que la tendencia constructivista en los profesores se relaciona con aquello que creen se debe hacer y no con aquello que creen hacer en sus clases. En este último aspecto, la evaluación muestra una fuerte tendencia tradicional. Estos resultados tienen implicancias en la formación inicial y continua del profesorado de ciencias, sobre todo en contextos de desarrollo profesional y carrera docente.
\end{abstract}

Palabras clave: pensamiento pedagógico; creencias curriculares; enseñanza de las ciencias; formación de profesores

\section{Pedagogical Thinking in Science Education. Analysis of Curricular Beliefs and their Implications for High School Teacher Training}

\begin{abstract}
The aim of this work was to study the pedagogical thinking of a set of 303 Chilean teachers who teach science, by analyzing the curricular beliefs and curricular action beliefs of the teachers. This study is part of a wider investigation (FONEDCYT 11130150) on the training prospective teachers whose background thinking, knowledge and practice of Chilean teachers who teach science in secondary level education. The information collected through a Likert-type questionnaire and treated with multivariate techniques shows that the constructivist trend of the teachers is related to what they think that it should be done and not to what they believe to do in their classes. In the latter aspect, the evaluation shows a strong traditional tendency. These results have implications on initial and continuing training of science teachers, especially in the context of professional development and teaching career.
\end{abstract}

Keywords: pedagogical thinking; curricular beliefs; science teaching; teacher formation 


\section{INTRODUCCIÓN}

En la formación de profesores existen varios factores a considerar al momento de analizar la relación entre la calidad de la educación y el desempeño profesional (Murillo, 2006). Estos factores han repercutido en muchos contextos educativos y por cierto en la enseñanza de la ciencias. Esto se hace notorio, cuando la innovación curricular no ha llegado a implementarse en las aulas y la práctica sigue atrapada en modelos que tienden a perspectivas tradicionales de enseñanza (Contreras, 2010; Murillo, 2006). En esta línea, y aunque los resultados de la evaluación docente muestra que más de un $70 \%$ de nuestros profesores es competente o destacado respecto a organización de la unidad, estructura y ambiente de la clase, en otros (Docentesmas, 2013), dichos resultados poco se relacionan con los logros alcanzados por los estudiantes. Al respecto, las investigaciones recientes muestran la necesidad de tratar la relación entre las competencias docentes y el desarrollo de las competencias básicas en los estudiantes (De Juanas, Ezquerra, Martín del Pozo y Pesquero, 2012).

En la misma línea, la prueba INICIA muestra que los futuros profesores de ciencias, se encuentran muy por debajo de los estándares esperados. En las carreras de pedagogía en ciencias (matemática, biología, física y química) solo el $7 \%$ de los futuros profesores que rindieron la prueba de conocimientos disciplinares alcanza un nivel sobresaliente (2012). Luego, si observamos las especialidades en ciencias experimentales estos niveles de insuficiencia aumentan a un promedio de $74 \%$ en conocimiento disciplinar, donde el $55 \%$ tiene además un nivel solo aceptable en los conocimientos pedagógicos.

La tendencia en estos resultados se puede atribuir a diversos aspectos, entre ellos el evidente bajo conocimiento disciplinar de profesores y futuros profesores, pero un aspecto sobre el cual ha versado la discusión e investigación, es el referido al pensamiento sobre cómo enseñar los contenidos de las disciplinas de especialización (Garritz, 2014; Friedrichsen, van Driel y Abell, 2011). Como proponen Pérez Gómez y Gimeno (1992), un aspecto sobre el cual hay que detener la atención es el pensamiento pedagógico. Un componente importante de este pensamiento son las creencias, las cuales se relacionan con la toma de decisiones para la práctica y permiten interpretar diversos aspectos, tales como la planeación, la selección de contenidos, la evaluación, formas en que los profesores implementan las reformas, etc. (Garritz, 2014; Grossman, 1990). Al respecto, la Didáctica de las Ciencias y sus numerosas investigaciones, señalan que las creencias de los profesores deben ser tratadas en la formación del profesorado, tanto inicial como continua(Bryan, 2003; Markic, Eilks y Valanides, 2008; Markic y Eilks, 2010; Martínez Aznar, Martín del Pozo, Rodrigó, Varela, Fernández y Guerrero, 2001, 2002; Porlán y Rivero, 1998; Shulman, 2005; So y Watkins, 2005; Wallace y Kang, 2004).En esta línea, retomando la experiencia chilena y sus resultados, podemos decir que se han instalado diversos dispositivos (proyectos, recursos e instrumentos de medición) y se han cambiado las formas de pensar la enseñanza y el aprendizaje, lo cual ha significado cambiar la escuela y mejorar los procesos (Pozo, Scheuer, Pérez Echeverría, Mateos, Martín y de la Cruz, 2006), sin embargo, un conjunto importante de profesores y futuros profesores siguen pensando y trabajando desde modelos poco deseables y/o con escasos niveles de logro y desempeño, poniendo en evidencia las visiones dominantes y tradicionales que hay con respecto a la ciencia, su enseñanza y su aprendizaje. Para ello las propuestas han sido diversas e innovadoras, centrándose en los procesos formativos iniciales de profesores, se desarrollan estrategias que promuevan el cambio y evolución tanto del pensamiento como del conocimiento más profesional de la labor docente (Contreras, 2015; Ezquerra, Rodríguez y Hamed, 2013; Ezquerra, Azcarate, Rivero, Hamed, Martín del Pozo y Solis, 2013; Ezquerra, De Juanas y Martín del Pozo, 2015).

En este contexto, desarrollamos y presentamos parte de una investigación más amplia (FONDECYT 11130150), que tiene como antecedentes precisamente el pensamiento, conocimiento y práctica de profesores chilenos que enseñan ciencias en educación media. En otras palabras, el trabajo que presentamos corresponde a antecedentes empíricos, que fundamentan y relevan la importancia de tratar aquello relacionado con el pensamiento pedagógico, abordándolo desde sus elementos más específicos y a la vez cotidianos, como lo son los aspectos curriculares de contenidos, metodología y evaluación (pensamiento pedagógico-curricular). Así, esta investigación supone un acercamiento a las creencias curriculares y creencias de actuación curricular, como así también un acercamiento a la consistencia entre estas, con la idea además de mostrar las implicancias que las creencias curriculares tienen en la práctica y, por lo tanto, en la formación del profesorado. En definitiva, nos preguntamos ¿qué pensamiento pedagógico-curricular presentan los profesores en activo de ciencias chilenos? ¿Este pensamiento es coherente para los aspectos pedagógico-curriculares de contenidos, metodología y evaluación?

\section{CREENCIAS CURRICULARES ASOCIADAS A LA ENSEÑANZA DE LAS CIENCIAS}

La investigación señala que en los profesores existe un conjunto frecuente de creencias y creencias de actuación curricular, con respecto a qué enseñar (contenidos), cómo enseñar (metodología) y, qué y cómo 
evaluar (evaluación).En relación al aspecto curricular de contenidos los profesores creen importante el dominio conceptual según la lógica de la disciplina, resaltando el método científico. Ello pone de manifiesto las epistemologías de los profesores, con respecto a la naturaleza del contenido escolar (Chang, 2003; Martín del Pozo, 2001; Sánchez y Valcárcel, 2000). Sin embargo, una minoría da importancia a los contenidos procedimentales (García-Ruiz y Orozco, 2008; Van Driel, Bulte y Verloop, 2005). Esta tendencia más fragmentada, también se ha encontrado respecto al uso de ejemplos de la vida cotidiana como contenidos para establecer relaciones y explicar. Los profesores suelen utilizar dichos ejemplos, pero como un recurso para motivar (Abd-El-Khalick, 2005; Bryan, 2003; Cheng, Chan, Tang y Cheng, 2009; So y Watkins, 2005). Lo anterior, se relaciona con aspectos importantes del conocimiento y pensamiento sobre el contenido curricular a enseñar, como lo son su selección, su secuenciación y su organización (García-Ruiz y Orozco, 2008; Richoux y Beaufils, 2003).Por ejemplo, se ha señalado que lo profesores elaboran propuestas curriculares en las cuales los contenidos conceptuales son muy diversos, presentan pocas relaciones, están jerarquizados y las secuencias son lineales y acumulativas (So y Watkins, 2005).

Respecto de cómo enseñar, se ha encontrado que los profesores tienden a unir actividades, explicación y aprendizaje. De esta forma, la metodología es concebida como una secuencia única y cerrada de actividades, que se planifica para saber qué recursos y/o materiales utilizar (Richoux y Beaufils, 2003; Wallace y Kang, 2004). De hecho, las actividades son consideradas como un vehículo para llegar a los contenidos y, por lo tanto, centradas en los conceptos y en la comprobación de la teoría (Azcarate y Cuesta, 2005; Martínez Aznar et al., 2001, 2002). Por lo tanto, y pese a la posible existencia de otros materiales, recursos y actividades, los más usados son la pizarra, el libro de texto, listado de ejercicios y prácticas de laboratorio (González, Martínez Losada y García, 2006).

Sobre la evaluación, la evidencia es más tajante al señalar una tendencia marcadamente tradicional en las creencias de los profesores, donde el objetivo de evaluar es comprobar el aprendizajes de conceptos, los instrumentos son las pruebas o exámenes iniciales y finales, los cuales son diseñados por el profesor que imparte la asignatura (Azcarate y Cuesta, 2005; Garritz, 2014; Martínez Aznar et al., 2001, 2002; Solís, Luna y Rivero, 2002; Van Driel, Bulte y Verloop, 2005).

Por otro lado, y aunque la investigación ha considerado profesores de las diversas especialidades y trayectorias de experiencia profesional, tanto la evidencia cuantitativa como cualitativa, señala que no existen diferencias marcadas entre las distintas disciplinas o diferentes niveles de enseñanza. Al respecto, diversos investigadores señalan que este pensamiento sería similar al encontrado en futuros profesores (Abd-El-Khalick, 2005; Akerson, Morrison y McDuffie, 2006; Brown y Malear, 2006; Fernández y Tuset, 2008; Fernández, Tuset, Pérez y Leyva, 2009; Garritz, 2014; Markic et al., 2008; Markic y Eilks, 2010; Martínez Aznar et al., 2001, 2002; Van Driel, Bulte y Verloop, 2005).En otras palabras, profesores de primaria o secundaria, sin importar especialidad y/o experiencia, presentan similares creencias, en relación a los aspectos curriculares que venimos tratando, por lo tanto, dichos profesores, presentan un pensamiento similar, constituido por un conjunto de creencias compartidas a pesar de las múltiples diferencias que en tanto formación y experiencia han tenido (Contreras, 2010).

\section{METODOLOGÍA}

El estudio se realizó con un grupo de 303 profesores de enseñanza media de ciencias perteneciente a la Octava Región de Chile. Específicamente, se incorporó a profesores de las cuatro provincias: Concepción $(\mathrm{N}=141)$; Bio-Bio $(\mathrm{N}=54)$; Arauco $(\mathrm{N}=33)$ y Nuble $(\mathrm{N}=75)$. Todos ellos de ciencias experimentales (Biología, Física y Química) y/o matemáticas.

Recogimos la información de carácter cuantitativo, utilizado como instrumento un cuestionario tipo Likert $(1 \rightarrow 5)$ organizado en tres bloques, con la idea de explorar con qué creencias curricular y qué creencias de actuación curricular se identificaban los profesores. Para ello adaptamos y trabajamos con el cuestionario utilizado por Martínez Aznar et al. (2001 y 2002), con los respectivos procesos de pilotaje y validación de constructo y de contenido. El primer bloque contuvo los datos de identificación. El segundo bloque, integrado por 34 proposiciones sobre las creencias curriculares cuyas posibles respuestas fueron: totalmente de acuerdo (5), de acuerdo (4), indeciso (3), en desacuerdo (2) y totalmente en desacuerdo (1) y; un tercer bloque, también integrado por 34 proposiciones relacionadas con las creencias de actuación curricular, y cuyas posibles respuestas fueron: siempre (5), frecuentemente (4), a veces (3), casi nunca (2), nunca (1).

Por otro lado, utilizamos aquellas categorías curriculares que las investigaciones tratan, seleccionando aquellas categorías que estaban presentes en el instrumento de referencia y redefiniendo las subcategorías, según la teoría y la evidencia empírica (Markic y Eilks, 2010; Martínez Aznar et al., 2001, 2002). Estas categorías y subcategorías tuvieron diferentes significados según la tendencia curricular a la que nos 
refiramos: desde una más simple o tradicional hasta otra más compleja o constructivista, pasando por estadios intermedios o de transición (Fernández y Tuset, 2008; Fernández, Tuset y Leiva, 2009). En relación a las técnicas de análisis, y para la parte de los resultados cuantitativos que aquí presentamos, consideramos aquella técnica que nos permitiera discriminar entre las proposiciones del instrumento y cuyos resultados se consideraran significativos, pues en éstas proposiciones se reflejan la tendencia de una mayoría de los profesores. Nos referimos a la técnica multifactorial de componentes principales (Gil y Rico, 2003; Porlán, Martín del Pozo y Toscano, 2002), además el estadístico clásico de media. Por otro lado, y para determinar si existía relación entre el pensamiento (creencias curriculares) y acción (creencias de actuación curricular) aplicamos el test de $\mathrm{Chi}^{2}(0,0056<0.01)$ y test exacto de Fischer $(0,0055<0.01)$, además de los valores de KMO (0.81) para aplicar el análisis de componentes principales. Todo ello índico que existía una asociación fuerte entre creencias curriculares (pensamiento) y creencias de actuación curricular (acción). La probabilidad de ocurrencia de estos resultados, es menor que 0,01 y el nivel de confianza es mayor al $99 \%$. Es decir, la probabilidad de que las proposiciones que han resultado significativas y la relación entre pensamiento y acción sean producto del azar, es casi nula.

\section{RESULTADOS}

Obtuvimos dos factores para el cuestionario de creencias curriculares ( $P$ = pensamiento), dos para el cuestionario creencias de actuación curricular $(A=$ pensamiento sobre la actuación) y dos para el conjunto de las proposiciones $(P+A)$, los cuales explican un $26,1 \%, 24,8 \%$ y un $19,4 \%$ de la varianza, respectivamente. Las proposiciones seleccionadas y que conforman cada factor presentan un coeficiente de correlación (carga) superior a 0,5.

\section{Los factores que describen las creencias curriculares $(P)$}

Las proposiciones que configuran cada uno de los factores sobre el pensamiento se relacionan con los contenidos, la metodología y la evaluación (Tabla 1). Más concretamente, las del factor 1 están relacionadas con los contenidos y evaluación, el que explican el 15,3\% de la varianza total. Los profesores muestran su desacuerdo en considerar que el conocimiento científico es distinto al conocimiento escolar (0.527/2.4). Es decir, los profesores consideran que aquello que enseñan es conocimiento científico. Por otro lado, señalan que el objetivo de la evaluación es comprobar el nivel de conocimientos que tienen los alumnos (0.538/4.1), aunque lo más importante no sea necesariamente medir la adquisición de conceptos. El factor 2, explica un $10,8 \%$ de la varianza y se relaciona con las tres categorías estudiadas, mostrando una posición más constructivista. Los profesores se identifican con la conveniencia de usar una diversidad de actividades (0.598/4.7), fuentes de selección de contenidos (0.521/4.8), así como evaluar también procedimientos y actitudes $(0.601 / 4.5)$.

Tabla 1: Factores para creencias curriculares $(26.1 \%$ de la varianza)

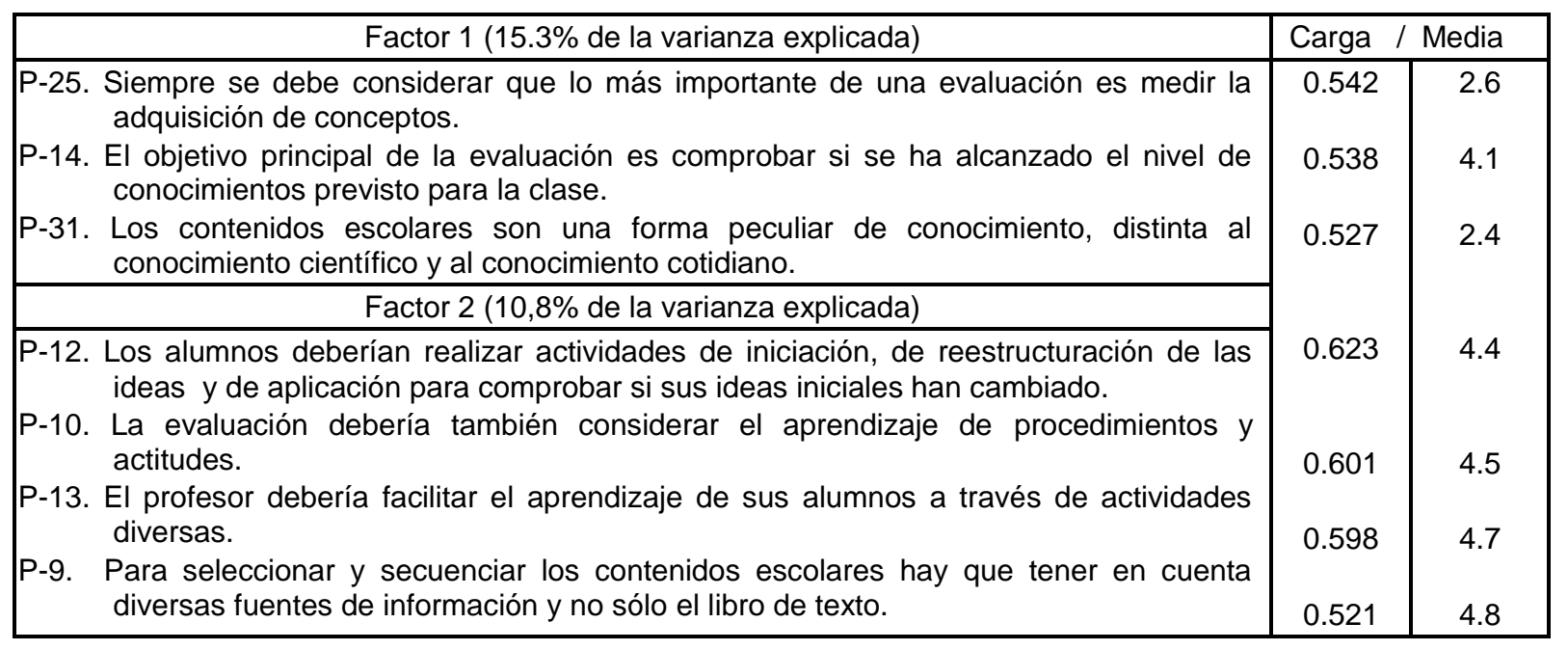

\section{Los factores que describen las creencias de actuación curricular $(A)$}

Las proposiciones que configuran los dos factores para las creencias de actuación curricular explican un $24,8 \%$ de la varianza y se relacionan con los contenidos, la metodología y la evaluación (Tabla 2). El factor 1 que explica el $13,4 \%$ de la varianza, señalando que los profesores se identifican con trabajar los aspectos históricos para enseñar ciencias, con lo cual ponen de manifiesto su carácter evolutivo y relativo (0.570/4). Por otro lado, indican organizar los contenidos en mapas, para relacionar unos contenidos con otros 
(0.545/4.1), considerando la utilidad práctica de éstos para motivar a los alumnos (0.529/4.2). Además, señalan dedicar atención específica a los alumnos con problemas y evaluar positivamente la evolución de ideas (0.619/3.6). Sin embargo, la mayoría considera que las actividades prácticas de laboratorio sirven para comprobar la teoría explicada en las clases (0.524/4.2). El factor 2 explica el 11,4\% de la varianza y describe a un grupo de profesores que señala utilizar a veces el libro de texto como fuente (0.533/3.3) y casi siempre como recurso para explicar y enseñar los contenidos (0.595/3.8). Por otro lado, se identifican son que frecuentemente las decisiones sobre la marcha de las clases son exclusivas del profesor (0.608/3.6). Además, tienden al uso del examen escrito porque es un instrumento objetivo (0.573/4), cuyo diseño es propio del profesor de la asignatura (0.523/3.8), es decir, individual.

Tabla 2: Factores para creencias de actuación curricular (24.8\% de la varianza)

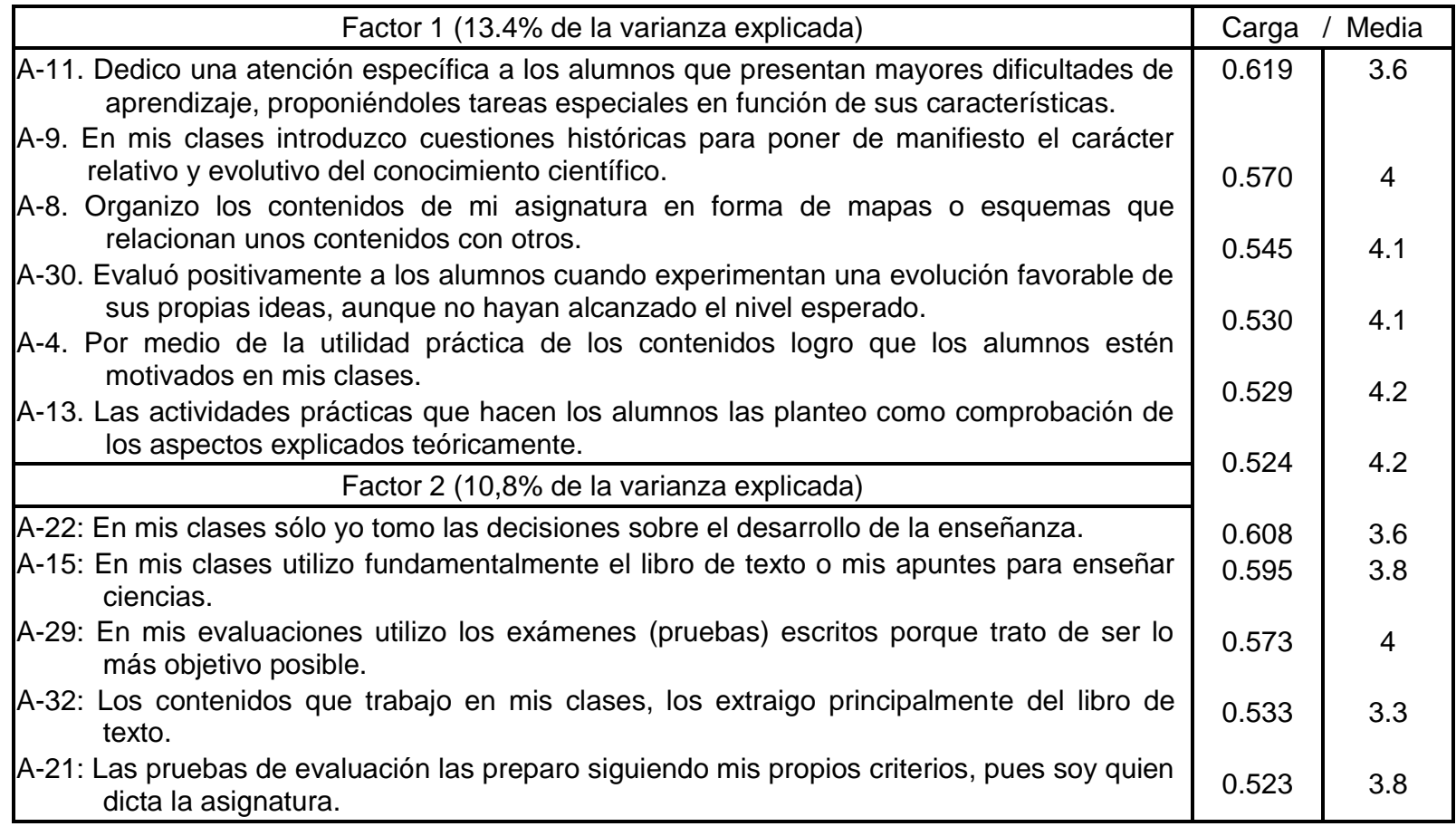

\section{Los factores que describen las creencias curriculares $(P+A)$}

Para el conjunto de las proposiciones sobre el pensamiento y la posible actuación obtuvimos dos factores que explican un $19,4 \%$ de la varianza (Tabla 3). El factor 1, señala que los profesores se muestran de acuerdo en considerar que el conocimiento científico es un conocimiento producto de teorías probadas $(0.546 / 3.8)$, pero no un conocimiento diferenciado del resto $(0.580 / 2.4)$, como por ejemplo, el escolar. Se muestran en desacuerdo en considerar que el libro de texto sea el recurso fundamental (0.571/2.7), no obstante indecisos respecto a usar utilizar el libro texto para seleccionar $(0.626 / 3)$ y explicar los contenidos a enseñar (0.645/2.8). Además, no consideran que la finalidad de la evaluación sea medir la adquisición conceptual $(0.654 / 2.6)$. Por otro lado, una mayoría prefiere planificar en lecciones y considera que adaptar la enseñanza perjudica a los más capacitados (0.574/3.6). En el factor 2, observamos que los profesores creen, en la práctica, utilizar frecuentemente diversos instrumentos para evaluar a sus alumnos, incluido el cuaderno de trabajo individual (0.552/3.8), también consideran evaluar procedimientos y las actitudes (0.529/4). Sin embargo, y aunque las proposiciones tienen un carácter más alternativo y cargas factoriales considerables, es insuficiente como para señalar un grupo de proposiciones con una tendencia constructivista, dada la varianza explicada (7.7\%).

\section{Consistencia entre creencias y creencias de actuación curricular}

Al realizar un análisis por aspectos pedagógico curricular, observamos que los profesores muestran inconsistencias entre sus creencias curriculares y sus creencias de actuación curricular, mostrándose más constructivistas en pensamiento y tradicionales en la actuación. Específicamente: (a) aunque los profesores consideran organizar el conocimiento en mapas, que incluyen aspectos históricos de la ciencias y de la utilidad práctica, solo se utilizan para motivar, pues el contenido escolar que se enseña sería igual al conocimiento científico; (b) para enseñar este conocimiento consideran que se debe utilizar diversas actividades y fuentes, no obstante, en la práctica, el libro de texto y las actividades prácticas de laboratorio son fundamentales; (c)consideran que se debe evaluar procedimientos, actitudes y la evolución de la ideas 
(evaluación), pero que el objetivo fundamental es siempre medir el nivel de conocimientos previstos. Para ello, utilizan frecuentemente un examen escrito que es elaborado con criterios propios y; (d) el análisis multifactorial del conjunto de las proposiciones $(P+A)$ muestra a un conjunto de profesores con una tendencia más homogénea sobre sus creencias curriculares, no así sobre sus creencias de actuación curricular. Ello indicaría, primero una inconsistencia entre aquello que creen se debe hacer y aquello que creen hacer. Además, de indicarnos posiblemente un pensamiento (constituido por creencias) más compartido y con una tendencia más constructivista, a diferencia de la actuación docente (Tabla 4).

Tabla 3: Factores que describen la relación $P+A(19.4 \%$ de la varianza)

\begin{tabular}{|c|c|c|}
\hline Factor 1 (11.7\% de la varianza explicada) & Carga & Media \\
\hline $\begin{array}{l}\text { P-22. Tener en cuenta la diversidad de los alumnos a la hora de impartir las materias de } \\
\text { ciencias periudica a los alumnos más capacitados. }\end{array}$ & 0.656 & 3.2 \\
\hline $\begin{array}{l}\text { P-25. Siempre se debe considerar que lo más importante de una evaluación es medir la } \\
\text { adquisición de conceptos. }\end{array}$ & 0.654 & 2.6 \\
\hline P-32. Cada tema debería explicarse siguiendo el libro de texto o los propios apuntes del & 0.645 & 2.8 \\
\hline $\begin{array}{l}\text { profesor. } \\
\text { P-23. Los libros de texto son la fuente de información fundamental para seleccionar los }\end{array}$ & 0.626 & 3 \\
\hline $\begin{array}{l}\text { contenidos que hay que enseñar. } \\
\text { P-31. Los contenidos escolares son una forma peculiar de conocimiento, distinta al }\end{array}$ & 0.580 & 2.4 \\
\hline conocimiento científico y al conocimiento cotidiano. & 0.574 & 3.6 \\
\hline P-27. Planifico mi enseñanza a partir de lecciones. & 0.571 & 2.7 \\
\hline $\begin{array}{l}\text { P-18. El libro de texto es el recurso fundamental para enseñar y aprender ciencias. } \\
\text { P-6. El conocimiento es producto de la acumulación de teorías que han sido comprobadas. }\end{array}$ & 0.546 & 3.8 \\
\hline \multicolumn{3}{|l|}{\begin{tabular}{|c|} 
Factor $2(7.7 \%$ de la varianza explicada $)$ \\
\end{tabular}} \\
\hline $\begin{array}{l}\text { A-2. Utilizo como parte de la evaluación final, la evaluación de los cuadernos de trabajo } \\
\text { individual y de los laboratorios. }\end{array}$ & 0.552 & 3.8 \\
\hline A-5. Cuando evaluó a los alumnos, considero además sus actitudes & 0.529 & 4 \\
\hline
\end{tabular}

Tabla 4: Tendencia de las creencias y creencias de actuación

\begin{tabular}{|c|c|c|c|c|c|c|c|}
\hline & \multicolumn{4}{|c|}{ Pensamiento $(\mathrm{P})$} & \multicolumn{3}{|c|}{ Acción $(A)$} \\
\hline & $\mathrm{T}$ & Interm & dia & C & $\mathrm{T}$ & Intermedia & C \\
\hline Contenidos & $P_{6}$ & $P_{31}$ & $\begin{array}{l}P_{23} \\
P_{31}\end{array}$ & $\mathrm{P}_{9}$ & & $\mathrm{~A}_{32}$ & $\begin{array}{l}A_{8} \\
A_{9}\end{array}$ \\
\hline Metodología & $\mathrm{P}_{27}$ & $P_{22}$ & $\begin{array}{l}P_{18} \\
P_{22} \\
P_{25} \\
P_{32}\end{array}$ & $\begin{array}{l}P_{12} \\
P_{13}\end{array}$ & $\begin{array}{l}A_{13} \\
A_{22}\end{array}$ & & $\begin{array}{l}A_{4} \\
A_{11}\end{array}$ \\
\hline Evaluación & $P_{14}$ & $\mathrm{P}_{25}$ & & $P_{10}$ & $\begin{array}{l}A_{15} \\
A_{29} \\
A_{21}\end{array}$ & & $\begin{array}{l}A_{2} \\
A_{5} \\
A_{30}\end{array}$ \\
\hline
\end{tabular}

Habiendo tratado solo con el pensamiento de los profesores y más específicamente con qué se identifican los profesores al pensar en su práctica, nos parece relevante que se presenten inconsistencias. Ello sugiere niveles de organización y articulación, dependiendo del aspecto pedagógico-curricular (en este caso) que se trate. Específicamente, primero un inconsistencia entre aquello que creen se debe hacer y aquello que creen hacer. Además, de indicarnos posiblemente un pensamiento (constituido por creencias) más compartido y con una tendencia más constructivista, a diferencia de la acción docente. Los profesores presentan o se identifican con una tendencia más constructivista en sus creencias curriculares que en sus creencias de actuación curricular, en relación a los contenidos, metodología y evaluación. Al tratarse de la práctica los profesores se identifican con creencias curriculares tradicionales. No obstante, también hemos encontrado unas tendencias intermedias en las que para una misma categoría curricular, los profesores se identifican con creencias constructivistas y tradicionales a la vez. En esta línea, y como indicáramos en otras investigaciones sobre el pensamiento del profesor respecto al aprendizaje (Contreras, 2010), las tendencias en el pensamiento y sus niveles de organización, muestran que los profesores se mueven entre una teoría directa y otra intermedia, sin llegar a una post-moderna (más constructivista), sin importar la especialidad.

\section{DISCUSIÓN}

El hecho de que una mayoría de los profesores considere que el contenido que enseña es conocimiento científico, nos muestra un desconocimiento respecto a qué se enseña, no desde el punto de vista disciplinar, sino más bien de la naturaleza del tipo de contenido, dado que no existe claridad en la diferencia entre el conocimiento científico y el contenido escolar (Chan, 2003; Martín del Pozo, 2001; Sánchez y 
Valcárcel, 2000). Coincidiendo con los resultados de otras investigaciones, aunque un número importante de profesores considera tratar y relacionar con los contenidos que enseña aspectos de la vida cotidiana, de utilidad práctica y de la historia, también la mayoría señala utilizarlos solo para motivar a los estudiantes (Bryan, 2003; So y Watkins, 2005). Esta posición más deseable, de relacionar unos contenidos con otros, se refiere al carácter más dinámico del conocimiento científico, donde los profesores relacionan los contenidos a enseñar con los factores sociales y culturales (Abd-El-Khalick, 2005; Cheng, Chan, Tang y Cheng, 2009).

Otro aspecto que es importante analizar y que se relaciona con los contenidos, es la tendencia a fragmentar y/o separar sus distintos tipos (conceptos, procedimientos, actitudes, etc.), resaltando casi siempre los contenidos conceptuales. De hecho, los profesores, señalan como importante tratar diversos tipos de contenidos y establecer relaciones entre ellos, por ejemplo a través de mapas conceptuales. No obstante, el conocimiento científico que es producto de teorías probadas es el que se debe enseñar. Esta posición ciertamente va en sentido contrario a aquel pensamiento donde se considera al conocimiento científico más dinámico. Al respecto, Chan (2003) señala que esto se debería a que los profesores no consideran que el contenido escolar sea un conocimiento con identidad epistemológica diferenciada. Esto tendrían efectos en la selección, secuenciación y organización de los contenidos (García-Ruiz y Orozco, 2008; Richoux y Beaufils, 2003). Además, diversos investigadores indican que este pensamiento sería similar al encontrado en futuros profesores (Abd-El-Khalick, 2005; Akerson, Morrison y McDuffie, 2006).

Un aspecto metodológico que llama la atención es aquel referido a los recursos. Los profesores consideran el uso de diversos recursos en diversas actividades, no obstante el libro de texto y las prácticas de laboratorio son fundamentales. So y Watkins (2005) señalan que desde estas posiciones, donde las propuestas curriculares presentan contenidos con pocas relaciones, jerarquías, secuencias acumulativas, además de un uso frecuente del libro de texto, indicarían que la metodología es concebida más bien como una secuencia única y cerrada de actividades (Wallace y Kang, 2004).De esta forma, las actividades no son consideradas como un proceso para lograr una comprensión adecuada de los contenidos, sino más bien, se cree que las actividades son vehículos, a través de los cuales, se puede llegar a los contenidos conceptuales y comprobarlos. Azcarate y Cuesta (2005), en sus investigaciones, señala que una mayoría de los profesores -de diversas disciplinas- cree utilizar diversas actividades, sin embargo, su selección depende de los contenidos. Estas actividades tienden a ser utilizadas con el fin de que los alumnos resuelvan problemas y comprendan los contenidos, además de comprobar la teoría. Todo ello también ha sido detectado en investigaciones con profesores de primaria y de secundaria (Fernández y Tuset, 2008; Fernández, Tuset, Pérez y Leyva, 2009; García-Ruiz y Orozco, 2008; Martínez Aznar et al., 2001, 2002).

Como se señala en el marco teórico, los profesores muestran una tendencia marcadamente tradicional respecto de la evaluación. Esta tendencia se da tanto en profesores como en futuros profesores, donde lo importante es comprobar y medir el nivel de los estudiantes (Azcarate y Cuesta, 2005). Según, Sánchez y Valcárcel (2000b) los profesores de secundaria, en sus evaluaciones, se limitan a tener en cuenta el nivel de conocimientos de los alumnos: cuánto saben los alumnos y cuánto deben saber. De hecho, y coherencia con esta tendencia más tradicional, entre los instrumentos más pensados y usados por los profesores, se encuentra el exámen escrito (Garritz, 2014; Martínez Aznar et a., 2001, 2002; van Driel, Bulte y Verloop, 2005). Todo ello coincide con nuestros resultados. De esta forma, aunque la competencia relacionada con el uso de la evaluación para informar a los alumnos sobre sus dificultades de aprendizaje, por ejemplo, es muy valorada (Solís, Luna y Rivero, 2002), parece ser una creencia de actuación poco extendida. A pesar de que la competencia docente de evaluación es altamente valorada cuando se trata de desarrollar competencias en y se deben evaluar todas las áreas curriculares (De Juanas, Ezquerra, Martín del Pozo y Pesquero, 2012).

\section{CONCLUSIONES}

Los profesores se identifican con creencias más constructivistas sobre las fuentes para el contenido, el uso de distintas actividades y recursos. En cambio, con creencias marcadamente tradicionales en la evaluación.

En términos multifactoriales, en su conjunto las proposiciones describen el pensamiento que presentan una tendencia más constructivista a diferencia de aquellas que describen la acción, y la relación que se describe entre pensamiento y posible práctica tiende a lo tradicional.

Aunque los profesores se identifican con una tendencia cercana a la constructivista en los aspectos curriculares de la enseñanza de las ciencias, esta tendencia está más relacionada con aquello que los profesores creen que se debe hacer y no con aquello que los profesores creen hacer en sus clases. Por lo tanto, existen incoherencias (inconsistencias) en el pensamiento del profesor. 
Consideramos que estos resultados tiene implicaciones para la formación inicial y continua de los profesores de ciencias, dado que estas investigaciones permiten de forma efectiva abordar una parte importante del conocimiento profesional de profesores y futuros profesores para mejorarlo, el pensamiento pedagógico, en este caso pedagógico-curricular. Por otro lado, también consideramos que estos resultados dan indicios importantes hacia dónde dirigir nuestra atención, sobre todo en un contexto de desarrollo y carrera profesional docente. Un acercamiento como el expuesto, indica niveles de organización y probablemente de desarrollo, que estarían ligados al conocimiento experencial, y que además probablemente dista de aquel pensamiento y conocimiento que futuros profesores puedan tener. Ello pone de manifiesto la necesidad de considerar el desarrollo de competencias profesionales básicas, utilizando estrategias que cuestionen el pensamiento de los profesores (futuros y en activo) y que promuevan su desarrollo y evolución, con la idea de innovar en la práctica y para la práctica.

\section{AGRADECIMIENTOS}

Fondo Nacional de Desarrollo Científico y Tecnológico (FONDECYT), CONICYT, Ministerio de Educación. Programa FONDECYT-INICIACION 2014, (Proyecto 11130150).

\section{REFERENCIAS}

Abd-El-Khalick, F., Developing deeper understandings of nature of science: the impact of a philosophy of science course on preservice science teachers' views and instructional planning. International Journal Science Education: 27 (1), 15-42 (2005)

Akerson, V., J. Morrison y A. McDuffie, One course is not enough: preservice elementary teachers' retention of improved views of nature of science. Journal of Research in Science Teaching: 43 (2), $194-213$ (2006)

Azcarate, G. y F. Cuesta, El profesorado novel de secundaria y su práctica. Estudio de un caso en las áreas de ciencias. Enseñanza de las Ciencias: 23 (3), 393-402 (2005)

Brown, S. Y C. Melear, Investigation of secondary science teachers' beliefs and practices after authentic inquiry-based experiences. Journal of Research in Science Teaching: 43 (9), 938-962 (2006)

Bryan, L., Nestedness of beliefs: examining a prospective elementary teachers' belief system about science teaching and learning. Journal of Research in Science Teaching: 40(9), 835-868 (2003)

Chan, K-W., Hong Kong teacher education students' epistemological beliefs and approaches to learning. Research in Education: 69 (1), 36 - 50 (2003)

Cheng, M., K-W Chan, S. Tang y A. Cheng, Pre-service teacher education students' epistemological beliefs and their conceptions of teaching.Teaching and Teacher Education: 25 (2), 319 - 327 (2009)

Contreras, S., Las creencias curriculares de los profesores de ciencias. Una aproximación a las teorías implícitas sobre el aprendizaje. Horizontes Educacionales: 15(1), 23-36 (2010)

Contreras, S., Flexible training models: a response to the current needs. Analysis of the teaching practices of chemistry. Generating an innovative component in the initial teaching training from the school. Procedia Social and Behavioral Sciences: 196, 30 -34 (2015)

Docentesmas. http://www.docentemas.cl/dm06_anuario2012.php. Acceso: junio (2015)

Ezquerra, A., F. Rodríguez y S. Hamed, Evolution of knowledge of future primary teachers: an education proposal using inquiry-based science. Procedia Social and Behavioral Sciences: 116, 1309-1313 (2013)

Ezquerra, A., P. Azcarate, A. Rivero, S. Hamed, R. Martín del Pozo y E. Solis. La formación inicial de maestros de primaria: qué hacer y cómo en didáctica de las ciencias. Enseñanza de las ciencias, Numero Especial, IX Congreso Internacional sobre investigación en didáctica de Is ciencias. 3045-3050 (2013)

Ezquerra, A., A. De Juanas y R. Martín del Pozo, Estudio sobre las actividades llevadas a cabo en la práctica docente universitaria para la formación inicial del profesorado de primaria y secundaria. Revista de Curriculum y formación del profesorado. 19(1), 330-345 (2015) 
Fernández, M., y A. Tuset, Calidad y equidad de las prácticas educativas de maestros de primaria mexicanos en sus clases de ciencias naturales. Revista Iberoamericana sobre Calidad, Eficacia y Cambio en Educación, 6(3), 156-171 (2008)

Fernández, M., A. Tuset, R. Pérez y A. Leyva, Concepciones de los maestros sobre la enseñanza y el aprendizaje y sus prácticas educativas en clases de ciencias naturales. Enseñanza de las Ciencias: 27(2), 287-298 (2009)

Friedrichsen, P., J. Van Driel y S. Abell, Taking a closer look at science teaching orientations. Science Education: 95(2), 358-376 (2011)

García-Ruiz, M., y L. Orozco, Orientando un cambio de actitud hacia las ciencias naturales y su enseñanza en profesores de educación primaria. Revista Electrónica de Enseñanza de las Ciencias: 7(3), 539-568 (2008)

Garritz, A., Creencias de los profesores, su importancia y cómo obtenerlas. Educación Química: 25(2), 88$92(2014)$

Gil, F., y L. Rico, Concepciones y creencias del profesorado de secundaria sobre enseñanza y aprendizaje de las matemáticas. Enseñanza de las Ciencias: 21(1), 27-47 (2003)

González, C., C. Martínez Losada y B. García, ¿Cuál es la secuencia de enseñanza del profesor de Ciencias? En Educación Científica: Tecnologías de la Información y la Comunicación y Sostenibilidad. XXII Encuentros de Didácticas de las Ciencias Experimentales, 13 a 16 de septiembre. Sesión VII. Zaragoza (2006)

Grossman, P., The making of a teacher: Teacher knowledge and Teacher education. New York: Teachers College (1990)

Markic, S., L. Eilks y N. Valanides, Developing a tool to evaluate differences in beliefs about science teaching and learning among freshman science students teachers from different science teaching domains: A case study, Eurasia Journal of Mathematics, Science \& Technology Education: 4(2), 109-120 (2008)

Markic, S., y L. Eilks, First-Year Science Education Student ness: Parallels and differences between chemistry and other science teaching domains, Journal of Chemical Education: 87(3), 335-339 (2010)

Martín del Pozo, R., Prospective teachers' ideas about the relationships between concepts describing the composition of matter. International Journal Science Education: 23 (4), 353 - 371 (2001)

Martínez Aznar, M., R. Martín del Pozo, V. Rodrigó, M. Varela, M. Fernández y S. Guerrero, ¿Qué pensamiento profesional y curricular tienen los futuros profesores de ciencias de secundaria? Enseñanza de las Ciencias: 19(1), 67-87 (2001)

Martínez Aznar, M., R. Martín del Pozo, V. Rodrigó, M. Varela, M. Fernández y S. Guerrero, Un estudio comparativo sobre el pensamiento profesional y la "acción docente", de los profesores de ciencias de educación secundaria. Parte II. Enseñanza de las Ciencias:20(2), 243-260 (2002)

Moreno, M. y C. Azcarate, Concepciones y creencias de los profesores universitarios de matemáticas acerca de la enseñanza de las ecuaciones diferenciales. Enseñanza de las Ciencias: 21(2), 265-280 (2003)

Moreno, M., El pensamiento del profesor. Evolución y estado actual de las investigaciones. En: Perafán, G.A. y Adúriz-Bravo, A. (Comps.). Pensamiento y conocimiento de los profesores. Debate y perspectivas internacionales, 127-139. Santafé de Bogotá: Universidad Pedagógica Nacional/Colciencias (2002)

Murillo, F., La formación de docentes: Una clave para la mejora educativa. Modelos innovadores en la formación inicial docentes. UNESCO. Santiago de Chile. 11-18 (2006)

Pérez Gómez, A., y J. Gimeno, El pensamiento pedagógico de los profesores: un estudio empírico sobre la incidencia de los cursos de aptitud pedagógica $(C A P)$ y de la experiencia profesional en el pensamiento de los profesores. Investigación en la Escuela: 17, 51-73 (1992)

Porlán, R., yA. Rivero, El conocimiento profesores. Sevilla: Díada Editora (1998) 
Porlán, R., R. Martín del Pozo y J. Toscano, Conceptions of school-based teacher educators concerning ongoing teacher Education. Teaching and Teacher Education: 18(3), 305-321 (2002).

Pozo, J., N. Scheuer, M. Mateosy M. Pérez Echeverría, Las teorías implícitas sobre el aprendizaje y la enseñanza. En: Pozo, Scheuer, Pérez Echeverría, Mateos, Martín y de la Cruz (Eds.): Nuevas formas de pensar la enseñanza y el aprendizaje. Barcelona: Grao (2006)

Richoux, H., D. y Beaufils, La planificación de las actividades de los estudiantes en los trabajos prácticos de física: análisis de prácticas de profesores. Enseñanza de las Ciencias: 21(1), 95-106 (2003)

Sánchez, G., y M. Valcárcel, ¿Qué tienen en cuenta los profesores cuando seleccionan el contenido de enseñanza? Cambios y dificultades tras un programa de formación. Enseñanza de las Ciencias: 18 (3), 423-437 (2000)

Shulman, L., Knowledge and teaching: Foundations of the New Reform. Harvard Educational Review: 57(1), 1-22 (2005)

So, W., y D.A. Watkins, From beginning teacher education to professional teaching: a study of the thinking of Hong Kong primary science Teachers. Teaching and Teacher Education: 21(5), 525-541 (2005)

Solís, E., M. Luna y A. Rivero,Las concepciones y los problemas profesionales del profesorado "novel" de secundaria del área de ciencias de la naturaleza. Demandas para la formación inicial. Fuentes: 4, 153 - 166 (2002)

Van Driel, J., M. Bulte y N. Verloop, The conceptions of chemisttry teachers about teaching and learning in the context of a curriculum innovation. International Journal of Science Education: 27(3), 303-322 (2005)

Wallace, C., y N-H. Kang, An investigation of experienced secondary science teacher' beliefs about inquiry: an examination of competing belief sets. Journal of Research in Science Teaching: 41(9), 936-960 (2004) 